Irish Math. Soc. Bulletin

Number 72, Winter 2013, 33-34

ISSN 0791-5578

\title{
UNIVERSALITY AND POTENTIAL THEORY
}

\author{
MYRTO MANOLAKI
}

This is an abstract of the $\mathrm{PhD}$ thesis Universality and potential theory written by Myrto Manolaki under the supervision of Prof. Stephen J. Gardiner at the School of Mathematical Sciences, University College Dublin, and submitted in May 2013.

Universality is an abstract notion which relates to various mathematical contexts. Generally speaking, an object is called universal if, via a countable process, it can approximate every object in some class. This thesis investigates one of the most widely-studied instances of universality: the case of universal series, where the approximation is obtained by considering subsequences of partial sums. In particular, it focuses on the study of harmonic functions on domains in $\mathbb{R}^{N}$, and holomorphic functions on planar domains, which possess universal polynomial expansions.

The first part of the thesis is concerned with the related phenomenon of overconvergence, which is of independent interest. Ostrowski showed that there are intimate connections between the gap structure of a Taylor series and the behaviour of its partial sums outside the disc of convergence. In Chapter 3 we investigate the corresponding problem for the homogeneous polynomial expansion of a harmonic function and we obtain analogues of the three main theorems of Ostrowski. We further show that the results for harmonic functions display new features in the case of higher dimensions.

Let $\Omega$ be a domain in $\mathbb{R}^{N}$ and let $w \in \Omega$. Chapter 4 is concerned with the class $U(\Omega, w)$ of harmonic functions on $\Omega$ which are universal about $w$, in the sense that subsequences of partial sums of the homogeneous polynomial expansion approximate arbitrary harmonic polynomials on arbitrary compact sets in $\mathbb{R}^{\mathbb{N}} \backslash \Omega$ that have

2010 Mathematics Subject Classification. 31B05, 30K05.

Key words and phrases. harmonic function; harmonic approximation; homogeneous harmonic polynomials; overconvergence; universal series.

Received on 14-12-2013.

Support from Science Foundation Ireland through the Research Frontiers Programme (project number 09/RFP/MTH2149) is gratefully acknowledged. 
connected complement. It is known that, if $\left(\mathbb{R}^{N} \cup\{\infty\}\right) \backslash \Omega$ is connected, then for each $w \in \Omega$, the class $U(\Omega, w)$ is non-empty. Under the assumption that $\Omega$ omits an infinite cone, we show that the connectedness hypothesis on $\left(\mathbb{R}^{N} \cup\{\infty\}\right) \backslash \Omega$ is essential, and that a harmonic function which is universal at one point is actually universal at all points of $\Omega$. Further, we conclude that no function in $U(\Omega, w)$ has a harmonic extension to a larger domain.

The other main component of the thesis is concerned with the class of holomorphic functions on planar domains which possess universal Taylor series. We adopt a potential theoretical approach in order to shed some light on the main questions of the area. In particular, we use various potential theoretical notions such as harmonic measure, the Martin boundary and minimal thinness, in order to address questions concerning the existence (Chapter 5) and the boundary behaviour (Chapter 6) of such functions. Our main result is that holomorphic functions possessing universal Taylor series are always unbounded.

Finally, some further work is presented in Chapters 7 and 8, which involve some results on potential theory and universal Laurent series.

The main results of this thesis are included in [1, 2, 3].

\section{REFERENCES}

[1] S. J. Gardiner and M. Manolaki, Boundary behaviour of universal Taylor series on multiply connected domains, submitted.

[2] M. Manolaki, Ostrowski-type theorems for harmonic functions, J. Math. Anal. Appl. 391 (2012), 480-488.

[3] M. Manolaki, Universal polynomial expansions of harmonic functions, Potential Anal. 38 (2013), 985-1000.

Department of Mathematics, University of Western Ontario, LonDON, ON N6A 3K7, CANADA

E-mail address, M. Manolaki: arhimidis8@yahoo.gr 